\title{
Forced Expiratory Volume in 6 Seconds
}

National Cancer Institute

\section{Source}

National Cancer Institute. Forced Expiratory Volume in 6 Seconds. NCI Thesaurus. Code C47843.

A test of lung function, the FEV 6 is the volume exhaled during six seconds of a forced expiratory maneuver started from the level of total lung capacity. 